\title{
Is the SIS 3.0 Valid for Use at a Rehabilitation Setting in Korea for Patients with Stroke?
}

\author{
Jumin Song, Haejung Lee \\ Department of Physical Therapy, College of Health \& Welfare, Silla University, Busan, Korea
}

Purpose: The purpose of this study was to assess psychometric properties of the Korean version of the Stroke Impact Scale 3.0 (K-SIS 3.0) in patients with stroke.

Methods: Patients with stroke longer than 3 months were invited to participate in the study at specialized rehabilitation centers in Busan. Information on patients was collected using Mini-Mental State Examination (MMSE), Modified Bathel Index (MBI), Beck Depression Index (BDI), WHODAS 2.0-12 item, and $K$-SIS. Floor and ceiling effects of each domain of $K$-SIS were examined. The internal consistency of each domain of the $K$-SIS was calculated using Cronbach's $\alpha$. Correlation between $K$-SIS and each scale was assessed using Spearman's correlation coefficient.

Results: Ninety subjects participated in the study. The $K$-SIS was found to have excellent internal consistency (Cronbach's $\alpha=0.93$ ). Each domain of the consistency ranged from 0.86 to 0.94 , except the emotion $(\alpha=0.51)$. Significant correlations were observed between MMSE and domains of memory and thinking, and communication ( $r=0.48$ and 0.52 respectively). BDI was negatively related to domains of emotion, ADL, mobility, and participation ( $r=-0.43,-0.49,-0.52$ and -0.33 respectively). Specific daily activity (MBI) and general functioning (WHODAS 2.0) were also found to be closely related to the domains of ADL, mobility, and participation (ranging from $r=-0.41$ to $r=-0.59$ ). No ceiling and floor effect was observed.

Conclusion: Excellent reliability and validity of $K$-SIS were obtained in the study and it could be suggested that $K$-SIS may be used for patients with stroke for collection of information on functioning in the clinical context.

Keywords: Stroke, Stroke impact scale, Quality of life

\section{서 론}

뇌졸중은 이동 능력, 일상생활동작, 의사소통 및 인지능력을 포함해 일상생활의 전반에 걸쳐 문제를 야기시켜 삶의 질에 영향을 미치는 대표적인 신경질환이다. 삶의 질은 개인의 신체적, 기능적, 심리적 및 사회적 건강을 다루는 다차원적 접근으로 이론적 프레임 워크는 ICF 에 기반하고 있다. ${ }^{2,3}$ 그러므로 뇌졸중 환자의 삶의 질에 대한 평가는 환자의 기능수행과 사회심리적 상태에 대하여 광범위하게 수집할 수 있으며, 적절한 치료계획과 적용 및 치료효과 측정에 도움이 된다.

삶의 질을 측정하는 많은 도구들이 설문형태의 환자-보고식으로 개발되어 사용되고 있다. Short Form 36과 Euro-Qol은 일반적인 건강 관련 삶의 질(health-related QOL, HRQOL)을 측정하는 도구로서 뇌 졸중뿐만 아니라 다양한 건강상태에서 사용되는 일반 HRQOL도구 이다. 반면 뇌졸중 영향 척도(Stroke Impact Scale, SIS), 뇌졸중 삶의 질
(Stroke-Specific Quality of Life) 및 Stroke Adapted Sickness Impact Profile은 뇌졸중 환자의 HRQOL을 제공하는 도구로써 ${ }^{4}$ 단기간 동안 뇌 졸중 환자의 건강 상태 변화를 측정하는 데 유용하다. ${ }^{5}$ SIS는 뇌졸중 환자의 삶의 질을 평가하는 도구로서 뇌졸중 환자의 건강과 관련된 생활 기능의 다양한 측면을 광범위하게 측정하기 위해 사용되고 있 다. ${ }^{6} \mathrm{SIS}$ 는 8 개의 영역 64 항목으로 구성되어, 전문가용, 대리인 및 자가 기입식의 3 가지 버전으로 개발되었다. 정서, 의사소통, 기억과 사고, 사회적 역할, 인지 등 8 개의 영역에서 뇌졸중으로 인한 삶의 질의 변 화의 측정에 타당도와 신뢰도가 높은 도구이며 ${ }^{7}$ 뇌졸중 영향의 평가 를 더 향상시키기 위해 SIS 3.0으로 개정되어 사용되고 있다. ${ }^{8}$

환자-보고식의 측정도구는 임상가와 관련 연구자들에 의해 사용 되며 임상 현장에서뿐만 아니라 환자의 일상생활 환경에서 환자가 수 행하는 것을 평가할 수 있기 때문에 더 가치가 있다. 뇌졸중 환자의 삶의 질을 객관적으로 측정하기 위해 선택되는 도구는 신뢰도와 타 
당도를 포함하여 측정도구로서의 특성이 확보되어야 한다. 신뢰도는 내적일치도와 검사-재검사를 사용하여 평가된다. 내적일치도는 측정 도구의 문항들에 대한 동질성의 정도를 의미하며, 검사-재검사 신뢰 도는 반복측정에 의한 신뢰도로서 환자의 상태변화가 없는 경우 두 측정값의 일치도를 나타낸다. 타당도는 연구자가 측정하고자 하는 개 념이나속성을 얼마나 정확하게 측정하고 있는 가를 의미한다. ${ }^{10}$

선행된 많은 국내 연구에서는 뇌졸중 중재 후 그 효과를 주로 폐 기능, 상지 기능, 보행 및 균형과 같은 신체 기능이나 일상생활동작과 같은 신체활동을 평가하는 도구를 사용하였다.11-14 또한 뇌졸중환자 의 QOL에 대한 연구는 중재 효과의 측정보다는 QOL에 영향을 주는 요인을 분석하는 연구가 대부분이었다.15 여러 나라 언어로 번역되어 가장 많이 사용되는 측정도구임에도 불구하고 우리나라의 임상에 서는 잘 사용되지 않고 있는 실정이다.16-19 따라서 본 연구는 뇌졸중 환자의 삶의 질을 평가하는 SIS 3.0 한국어판의 타당도를 검증하여 한국 임상에서의 활용 가능성을 확인하기 위해 수행되었다.

\section{연구방법}

\section{1. 연구대상}

본 연구는 2014년 6월부터 12월까지 부산광역시 소재 8 개의 재활병 원에서 뇌졸중으로 진단 받고 입원 치료 중인 90 명의 환자를 대상으 로 하였다. 대상자 선정조건은 유병기간이 급성기가 지난 3 개월 이상 인 환자와 한국형 간이정신 상태판별검사(mini-mental state examination- Korea version; MMSE-K) 결과 24점 이상으로 인지능력에 손상이 없는 환자이며, 본 연구의 내용을 이해하고 자발적으로 참여에 동의 한 환자를 대상으로 하였다.

\section{2. 실험방법}

1) 측정도구 및 방법

(1) 간이정신상태 판별검사(Mini-Mental State Examination, MMSE)

치매 진단과 인지기능 장애 유무를 판별하기 위해 개발된 MMSE는 신뢰도와 타당도가 높은 측정도구이다. 총 12 문항으로 지남력, 기억 등록, 기억회상, 주의 집중 및 계산, 언어 기능, 이해 및 판단 등 6 개 영 역으로 구성되어 있으 며 총 30점으로 24점 이상을 '확정적 정상, 19 점 이하를 '확정적 치매', 그리고 20-23점은 '치매 의심'으로 판별된다. 임상에서 뇌손상 환자의 인지수준을 평가하는 도구로 널리 사용되 고 있다. 본 연구에서는 한글판 MMSE를 사용하였다. ${ }^{20}$

\section{(2) 수정바델지수(Modified Barthel Index)}

일상생활동작의 자립도를 평가하기 위해 개발된 바델지수는 총 5 회 의 개정이 있었고 본 연구에서는 5 판을 한글판 수정바델지수를 사용
하였다.21 환자의 기능호전 변화를 즉각적으로 반영하는 평가도구로 일상생활동작 수행수준을 나타내는 신뢰도와 타당도가 높은 도구 이다. 개인위생, 목욕하기, 식사하기, 용변처리, 계단 오르기, 옷입기, 대변/소변 조절, 보행 또는 의자차 및 의사-침대 이동의 10 개 항목을 수행하는 동안 직접적인 관찰과 면접을 통하여 의존 정도를 각 항목 별로 5 단계의 점수로 표시하였다. 100점을 만점으로 $0-24$ 점은 완전 의존성, $25-49$ 점은 최대 의존성, $50 \sim 74$ 점은 부분 의존성, $75-90$ 점은 약간 의존성, 91-99점은 최소 의존성, 100점은 완전 독립을 나타낸다

\section{(3) Beck 우울척도 (Beck Depression Index, BDI)}

우울증을 선별하기 위해 개발된 BDI는 신뢰도와 타당도가 높은 자 가기입식 측정도구이다. 각 문항에서 0 점에서 3 점까지 총 63 점까지 분포하며 우울증의 인지적, 정서적, 동기적 및 신체적 증상 영역의 21 문항으로 구성되어 있으며 증상의 정도를 표현하는 구체적인 항목에 대한 답변으로 점수화 한다. 점수가 높을수록 우울 증상이 심함을 의미한다. 한글판 Beck 우울척도를 사용하였다. ${ }^{22}$

\section{(4) 세계보건기구장애 평가 목록 2.0 (WHODAS 2.0)}

ICF 프레임워크에 기초하여 만들어진 평가도구인 WHODAS 2.0는 인지, 이동, 자기 관리, 어울리기, 일상 활동 및 지역사회 참여의 6 가지 영역에서 기능수행 정도를 평가할 수 있으며 장. 단기 건강상태와 연 관된 일상생활수행능력을 측정할 수 있다. 최근 30 일동안 대상자가 각 항목을 수행하는 데 겪은 어려움에 대해 5 단계로 기입하였다. 어 려움이 없으면 0 점, 극도로 어렵거나 수행할 수 없으면 4점으로 최고 점수는 60 점이고 점수가 낮을수록 기능 수행 정도가 좋은 것을 의미 한다. ${ }^{23} \mathrm{WHODAS} 2.0$ 는 최근 한글로 번역되었으며, 한글도구에 대한 높은 신뢰도와 타당도가 확보되어있다. ${ }^{24}$

\section{(5) 뇌졸중 영향 척도 3.0 (Stroke Impact Scale 3.0)}

SIS 3.0 은 뇌졸중 환자의 삶의 질을 평가하기 위한 신뢰도와 타당도 가 높은 자가보고식 평가 도구이다. 뇌졸중의 전반적인 회복을 측정 하기 위해 근력, 손의 기능, 일상생활동작, 이동, 의사소통, 정서, 기억 과 사고 및 사회적 참여의 8 개 영역의 59 항목으로 구성되어 있다. 설 문지를 작성하기에 앞서 검사자는 대상자에게 지난 한 주 동안 느꼈 던 것을 근력에 대한 항목은 근육의 강한 정도를 답하게 하고 기억과 사고, 의사소통, 일상생활동작, 이동 및 손의 기능 항목은 어려움의 정도를 답하게 하였으며 정서와 사회적 참여는 빈도의 정도로 답할 것을 설명하였다. 점수는 5 (전혀 없다), 4 (약간 있다), 3 (다소 있다), 2 (매우 있다), 1 (극도의 있다)점으로 전체 점수가 높을수록 삶의 질 이 높은 것을 나타낸다. 정서 영역에서 6번 (이전과 같이 뭔가를 즐길 수 있음), 8 번 (삶이 가치가 있다고 느낌), 9번 (적어도 하루에 한 번 이 
상 미소 짓기) 항목은 다른 항목의 답변과 반대의 척도로 구성되어 있어 점수를 역으로 계산하여야 한다. ${ }^{8,25}$ 본 연구에서는 우리나라 실 정에 맞게 번역한 한글판 SIS 3.0 을 사용하였다. ${ }^{26}$

\section{2) 자료수집방법}

본 연구에 참여한 대상자들에게 연구에 대한 의의를 설명하였으며, 대상자 본인이나 대리인으로부터 연구에 대한 동의를 받았다. 데이 터 수집은 임상경험 3년 이상의 신경계 물리치료사가 실시하였으며, 본 연구를 위하여 상기의 측정도구 사용에 대해 교육을 사전에 실시 하였다. 대상자들의 이해도를 높이기 위해 물리치료사가 측정도구 항목을 읽고 환자의 답변을 기록하는 방식으로 진행되었다.

\section{3. 자료분석}

연구대상자의 일반적인 특성 및 각 측정 변수는 기술통계로 분석하 였다. 내적일치도는 Cronbach's a 값으로 분석하였으며, 타당도는 스피 어만 상관분석을 이용하여 SIS의 각 영역과 K-MMSE, K-MBI, K-BDI 및 K-WHODAS과의 상관관계를 측정하였다. 또한 K-SIS 3.0의 각 영역 에 대한 바닥효과와 천정효과를 관찰하였다. 통계프로그램은 IBM SPSS 21 버전을 사용하였으며, 통계학적 유의수준은 0.05 로 하였다.

\section{결 과}

\section{1. 대상자의 일반적 특성}

발병이 3개월 이상인 90명 (남성 60명, 여성 30명)의 뇌졸중 입원환자 가 본 연구에 참여하였다. 대상자 연령의 평균은 $52.7 \pm 15.6$ 세였다. 뇌 졸중의 유형은 뇌출혈이 46 명, 뇌경색이 44 명으로 구성되었으며, 마

Table 1. The means and standard deviations of measured scales

\begin{tabular}{llc}
\hline Scale & mean \pm standard deviation \\
\hline K-SIS & Strength & $9.48 \pm 3.52$ \\
& Memory \& Thinking & $29.21 \pm 5.89$ \\
Emotion & $30.70 \pm 5.52$ \\
Communication & $31.42 \pm 4.97$ \\
Activity & $34.46 \pm 9.62$ \\
Mobility & $28.61 \pm 10.07$ \\
Hand Function & $9.61 \pm 5.35$ \\
Participation & $20.90 \pm 9.50$ \\
Total & $194.17 \pm 38.05$ \\
K-MMSE & $28.02 \pm 3.82$ \\
K-MBI & $70.23 \pm 24.30$ \\
K-BDI & $40.86 \pm 12.15$ \\
K-WHODAS 2 & $82.97 \pm 46.46$
\end{tabular}

K-SIS, Korean version of Stroke Impact Scale 3.0; K-MMSE, Korean version of Mini-Mental State Examination; K-MBI, Korean version of Modified Barthel Index; K-BDI, Korean version of Beck Depression Index; K-WHODAS 2.0, Korean version of World Health Organization Disability Assessment Schedule.
비 측은 오른쪽이 45 명, 왼쪽이 45 명으로 구성되었다.

\section{K-SIS의 각 영역과 기존 도구의 기술통계량}

K-SIS의 각 영역과 K-MMSE, K-MBI, K-BDI 및 K-WHODAS 2.0의 기 술통계량은 Table 1에 나타내었다.

\section{K-SIS의 천정효과와 바닥효과}

임상측정도구는 최대점수와 최소점수의 비율이 전체 $15 \%$ 를 넘지 않 아야한다. 기억과 사고 및 의사소통영역에서는 천정효과가 관찰되었 으며, 이를 제외한 영역과 K-SIS의 총점에서는 천정효과와 바닥효과 는 관찰되지 않았다(Figure 1).

\section{K-SIS의 내적일치도}

감정영역을 제외한 영역에서 매우 높은 내적일치도가 관찰되었다. 정 서영역을 구성하는 항목 간의 동질성이 낮은 것으로 관찰되었다( $a$ $=0.56)$ (Table 2$)$.

\section{K-SIS와 기존 도구의 상관성}

K-SIS의 각 영역과 K-MMSE, K-MBI, K-BDI 및 K-WHODAS 2.0의 상 관관계 결과는 Table 3 에 나타내었다. 기억과 사고 영역 및 의사소통 영역에서는 K-MMSE의 항목과 활동영역 및 이동영역은 K-MBI와 높 은 연관성이 관찰되었다. K-BDI는 정서영역뿐만 아니라 활동영역 및 이동영역에서도 서로 높은 음의 상관관계를 나타내었다. 활동영역 및 이동영역은 또한 전반적인 기능수행 정도를 나타내는 K-WHODAS 2.0와매우 높은 음의 상관관계가 관찰되었다(Table 3).

\section{고 찰}

본 연구는 뇌졸중환자의 삶의 질을 다차원적으로 측정하는 도구인 K-SIS 3.0의 한국 임상영역과 관련 연구영역에서 그 활용가능성을 확

Table 2. The internal consistency of K-SIS of each domain

\begin{tabular}{lc}
\hline Domain of K-SIS & Cronbach's $\alpha$ \\
\hline Strength & 0.84 \\
Memory \& Thinking & 0.92 \\
Emotion & 0.56 \\
Communication & 0.91 \\
Activity & 0.91 \\
Mobility & 0.95 \\
Hand Function & 0.94 \\
Participation & 0.91 \\
Total & 0.82 \\
\hline
\end{tabular}

K-SIS, Korean version of Stroke Impact Scale 3.0. 

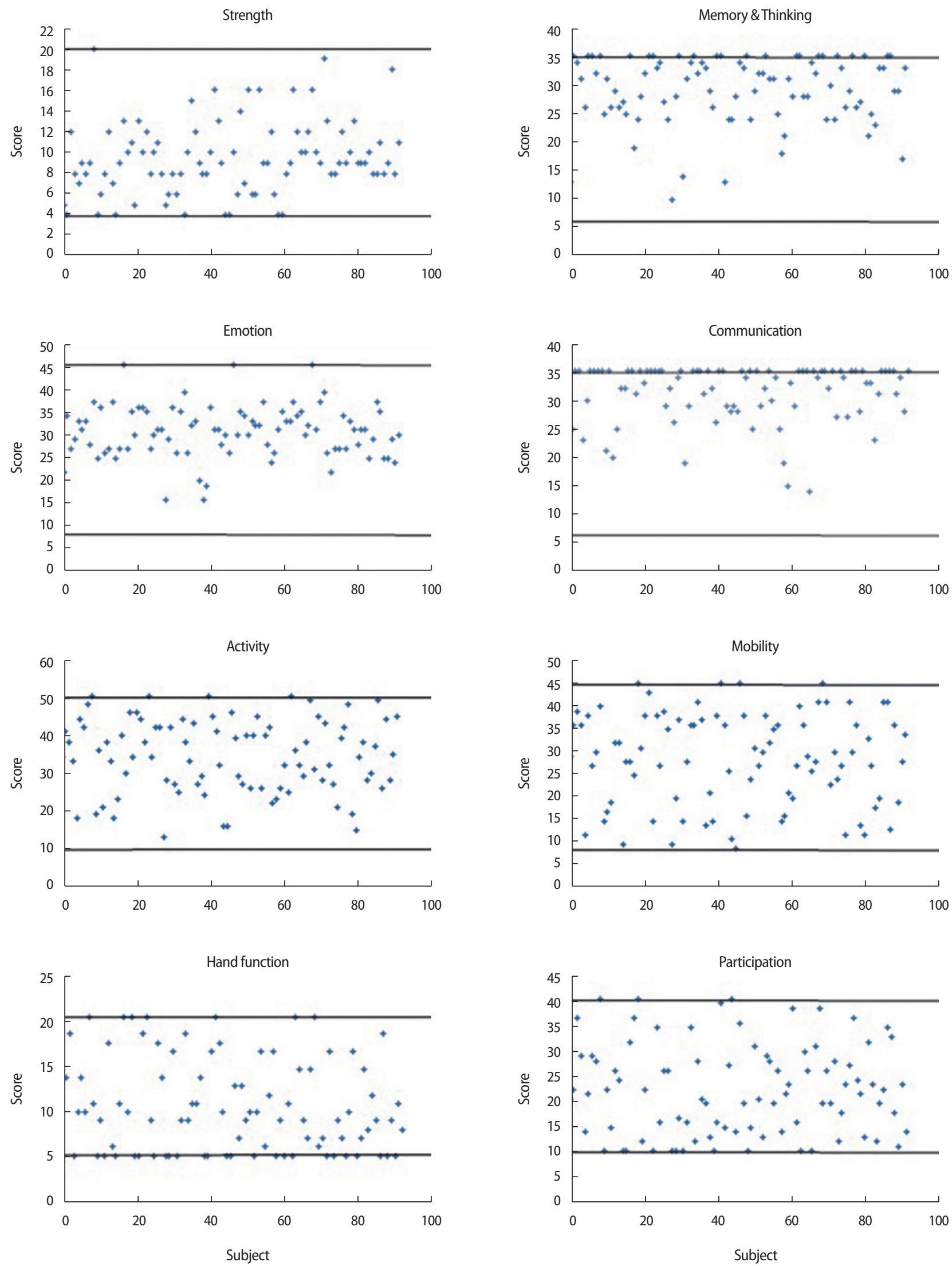

Figure 1. Ceiling and floor effect of each domain of K-SIS 3.0. 
Table 3. Correlation between each domain of K-SIS 3.0 and MMSE, $\mathrm{MBI}, \mathrm{BDI} \&$ WHODAS 2.0

\begin{tabular}{lcccc}
\hline K-SIS & K-MMSE & K-MBI & K-BDI & K-WHODAS 2.0 \\
\hline Strength & 0.19 & $0.34^{* *}$ & $-0.36^{* *}$ & $-0.43^{* *}$ \\
Memory \& Thinking & $0.49^{* *}$ & $0.41^{* *}$ & $-0.35^{* *}$ & $-0.28^{* *}$ \\
Emotion & $0.22^{*}$ & $0.34^{* *}$ & $-0.43^{* *}$ & $-0.44^{* *}$ \\
Communication & $0.52^{* *}$ & $0.29^{* *}$ & $-0.31^{* *}$ & $-0.27^{* *}$ \\
Activity & $0.34^{* *}$ & $0.61^{* *}$ & $-0.50^{* *}$ & $-.060^{* *}$ \\
Mobility & $0.28^{* *}$ & $0.66^{* *}$ & $-.052^{* *}$ & $-.062^{* *}$ \\
Hand Function & 0.17 & $0.37^{* *}$ & $-0.35^{* *}$ & $-0.42^{* *}$ \\
Participation & 0.18 & $0.40^{* *}$ & $-0.40^{* *}$ & $-0.46^{* *}$ \\
\hline
\end{tabular}

$* p<0.05 ; * * p<0.01$.

K-SIS, Korean version of Stroke Impact Scale 3.0; K-MMSE, Korean version of Mini-Mental State Examination; K-MBI, Korean version of Modified Barthel Index; K-BDI, Korean version of Beck Depression Index; K-WHODAS 2.0, Korean version of World Health Organization Disability Assessment Schedule.

인하기 위해 수행되었다. K-SIS 3.0에 대한 특성분석을 위하여 각 영역 의 항목 간 내적일치도와 K-MMSE, K-MBI, K-BDI 및 K-WHODAS 2.0 과의 상관관계를 이용한 타당도 분석을 실시하였다.

본 연구에 참여한 대상자는 전문재활병원에 입원하여 치료를 받 고 있는 만성 뇌졸중 환자들로서 인지기능의 손상이 없고 의사소통 이 원활한 수준의 환자들로 구성이 되었다. 참여한 환자들의 기능수 행수준은 선행연구에서와 유사하였다. ${ }^{16,18}$ 본 연구에서 감정영역에서 의 항목간 낮은 내적일치도는 SIS 원저를 포함한 선행연구에서 보고 된 결과와 비슷하다. ${ }^{8,16,18}$ 이는 정서 영역은 3 개의 긍정적 척도와 4 개 의 부정적 척도 항목이 혼합되어 구성되어 대상자들의 답변하는데 혼란이 야기될 수 있는 것으로 사료되며 이러한 측면이 낮은 내적일 치도에 영향을 미친 것으로 생각된다.

임상측정도구에서 최대점수와 최저점수의 비율이 전체의 $15 \%$ 를 넘으면 천정효과와 바닥효과의 존재를 의미한다. 천장효과가 있다면 환자의 상태가 더 호전되더라도 측정하지 못하며, 바닥효과는 환자의 상태가 악화되더라도 그 정도를 측정하지 못한다. 본 연구 결과 기억 과 사고영역 및 의사소통영역에서 천정효과가 관찰되었다. 선행연구 에서도 비슷한 결과를 보고하고 있다. ${ }^{16}$ 기억과 사고영역 및 의사소통 영역에서 나타난 천정효과는 인지기능과 의사소통에 문제가 없는 대 상자 선정으로 사료된다. 손기능 영역에서의 바닥효과 또한 선행된 연 구의 결과와 유사하다. ${ }^{16}$ 이는 뇌졸중 환자의 회복과정에서 손기능의 회복이 느리고 불완전하게 일어나는 것을 반영한 것으로 사료되며 손의 기능수행에 대한 정보를 정확하게 파악하고 싶다면 손의 기능 수행에 대해 특화된 도구를 사용하는 것이 타당할 것으로 생각된다.

SIS는 뇌졸중 환자의 건강상태와 관련하여 삶의 질을 평가하기 위 해 여러 영역에서의 기능수행 수준을 측정하는 데 사용되고 있 다. ${ }^{2,16,17,27} \mathrm{~K}-\mathrm{SIS}$ 의 활동영역 및 이동영역의 항목들은 일상생활동작을 평가할 수 있는 개념을 포함하고 있으며, 기억과 사고 영역 및 의사소
통 영역은 환자의 인지기능의 정도를 평가할 수 있는 것으로 본 연구 에서 확인하였다. 정서영역은 우울 정도를 측정하는 항목으로 구성 된 K-BDI와 높은 상관관계를 통하여 뇌졸중 환자의 정서상태에 대 한 정보를 확인하는 데 유용할 수 있을 것으로 사료된다. ${ }^{16,18}$ 흥미롭게 도 활동영역 및 이동영역에서도 K-BDI와 높은 상관관계가 관찰되었 다. 이는 정서적인 측면이 신체활동에 부정적으로 영향을 미치는 것 을 의미한다.22

활동영역, 이동영역 및 근력은 전반적인 기능수행 정도를 평가하 기 위한 항목으로 구성되어 있는 것을 관찰할 수 있었으며, 참여영역 은 각 도구들과 연관성이 나타나 참여 정도를 높이기 위해서는 인지 기능, 정서, 이동 및 활동능력 등의 수행력을 향상시키는 것이 중요하 다는 것을 알 수 있었다..$^{28}$ 본 연구의 결과 K-SIS가 뇌졸중 환자의 정보 를 근력과 인지기능을 포함한 신체기능뿐만 아니라 활동 및 참여 영 역까지 다면적 측면으로 측정하기에 적절한 도구임을 나타낸다. 본 연구는 연구 진행의 용이성을 위해 인지기능과 의사소통의 손상이 심각하지 않은 유병기간이 3개월 이상인 만성 뇌졸중 입원환자를 대 상으로 실시되어 연구의 결과를 일반화 하는데 제한점이 있다. 앞으 로의 연구는 K-SIS가 관련 분야에서 보편적으로 사용할 수 있도록, 만성기 뇌졸중 외래환자, 지역사회재활 대상 뇌졸중 환자 및 장애인, 시설수용 뇌졸중 장애인 등 다양한 특성의 대상자와 다양한 영역에 서 연구가 필요할 것으로 사료된다.

뇌졸중 환자의 삶의 질을 다차원적으로 측정하기 위해 개발된 뇌 졸중영향척도 3.0 의 한글판(K-SIS 3.0) 또한 항목간의 높은 내적타당 도와 기존의 표준 임상측정도구와 높은 상관관계가 관찰되어 한국 의 임상현장에서 환자의 삶의 질과 관련된 기능정보 수집을 위해 활 용하는데 적절한 도구라고 제안한다.

\section{REFERENCES}

1. Jönsson AC, Lindgren I, Hallström B et al. Determinants of quality of life in stroke survivors and their informal caregivers. Stroke. 2005;36(4): 803-8.

2. Duncan PW. Issues in selecting outcome measures to assess functional recovery after stroke. NeuroRx. 2006;3(4):505-24.

3. Song J, Lee H. Investigating functional level in patients with stroke using icf concept. J Kor Phys Ther. 2014;26(5):315-57.

4. Kranciukaite D, Rastenyte D. Measurement of quality of life in stroke patients. Medicina (Kaunas, Lithuania). 2006;42(9):709-16.

5. Vellone E, Savini S, Fida R et al. Psychometric evaluation of the stroke impact scale 3.0. J Cardiovascular Nursing. 2015;30(3):229-41.

6. Salter KL, Moses MB, Foley NC et al. Health-related quality of life after stroke: What are we measuring? Int J Rehab Res. 2008;31(2):111-7.

7. Duncan PW, Wallace D, Lai SM et al. The stroke impact scale version 2.0. Evaluation of reliability, validity, and sensitivity to change. Stroke. 1999; 30(10):2131-40. 
8. Duncan PW, Bode RK, Min Lai S et al. Rasch analysis of a new strokespecific outcome scale: The stroke impact scale. Arch Phys Med Rehabil. 2003;84(7):950-63.

9. Dawson J, Doll H, Fitzpatrick R et al. Routine use of patient reported outcome measures in healthcare settings. BMJ (Online). 2010;340 (7744):464-7.

10. Portney L, Watkins M. Foundations of clinical research: Applications to practice. 3rd. New Jersey, Pearson Education, 2009.

11. Kim J. Relationship between gait symmetry and functional balance, walking performance in subjects with stroke. J Kor Phys Ther. 2014; 26(1):1-8.

12. Yang D, Park S, Kang J et al. Effects of changes in postural alignment on foot pressure and balance of patients with stroke. J Kor Phys Ther. 2014; 26(4):226-33.

13. Yoon M, Choi H, Shin W. Effects of the abdominal drawing-in maneuver and the abdominal expansion maneuver on grip strength, balance and pulmonary function in stroke patients. J Kor Phys Ther. 2015;27(3): $147-53$.

14. Kim Y, Lee D. Effects of dance sports in virtual reality on balance, depression and adl in stroke patients. J Kor Phys Ther. 2013;25(5):360-36.

15. Kim H, Hwang Y, Yu J et al. The correlation between depression, motivation for rehabilitation, activities of daily living, and quality of life in stroke patients. J Korean Soc Occ Ther. 2009;17(3):41-53.

16. Carod-Artal FJ, Coral LF, Trizotto DS et al. The stroke impact scale 3.0: Evaluation of acceptability, reliability, and validity of the brazilian version. Stroke. 2008;39(9):2477-84.

17. Vellone E, Savini S, Barbato N et al. Quality of life in stroke survivors: First results from the reliability and validity of the italian version of the stroke impact scale 3.0. Annali di Igiene. 2010;22(5):469-79.

18. Geyh S, Cieza A, Stucki G. Evaluation of the German translation of the stroke impact scale using rasch analysis. Clin Neuropsychologist. 2009;
23(6):978-95

19. Mohammad AH, Al-Sadat N, Siew Yim L et al. Reliability and validity of the Nigerian (Hausa) version of the stroke impact scale (SIS) 3.0 index. BioMed Res Int. 2014;2014:302097.

20. Kwon Y, Park J, No I. Standardization of Korean of the mini-mental state examination (mmse-k) for use in the elderly. Part II. Diagnostic validity. Neuropsychiatric assoc. 1989;28(3):508-13.

21. Jung H, Park B, Shin H et al. Development of the Korean version of Modified Barthel Index (K-MBI): Multi-center study for subjects with stroke. J Kor Acad Rehabil Med. 2007;31(3):283- 97.

22. Hahn H, Yum T, Shin Y et al. A standardization study of beck depression inventory in Korea. J Korean Neuropsychiatry Assoc. 1986;25:487-502.

23. Garin O, Ayuso-Mateos JL, Almansa J et al. Validation of the "World Health Organization Disability Assessment Schedule, WHODAS-2.0" in patients with chronic diseases. Health and Quality of Life Outcomes. 2010;8.

24. Lee H, Kim D. Internal consistency and concurrent validity of korean language version of whodas 2.0: 12 item-self administered. J Kor Phys Ther. 2011;23(6):23-9.

25. Duncan PW, Lai SM, van Culin V et al. Development of a comprehensive assessment toolbox for stroke. Clin Geriatric Med. 1999;15(4):885915.

26. Lee H, Song J. The Korean language version of stroke impact scale 3.0: Cross-cultural adaptation and translation. J Kor Soc Phys Med. In press.

27. Hamza AM, Al-Sadat N, Loh SY et al. Predictors of poststroke healthrelated quality of life in nigerian stroke survivors: A 1-year follow-up study. BioMed Res Int. 2014;2014:350281.

28. Salter K, Jutai JW, Teasell R et al. Issues for selection of outcome measures in stroke rehabilitation: Icf participation. Dis \& Rehabil. 2005; 27(9):50728. 